\title{
MiR-200c sensitizes Olaparib-resistant ovarian cancer cells by targeting Neuropilin 1
}

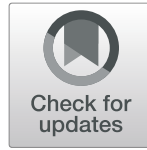

Enrica Vescarelli', Giulia Gerini', Francesca Megiorni', Eleni Anastasiadou1, Paola Pontecorvi ', Luciana Solito ', Claudia De Vitis ${ }^{2}$, Simona Camero ${ }^{3}$, Claudia Marchetti ${ }^{3}$, Rita Mancini ${ }^{2}$, Pierluigi Benedetti Panici ${ }^{3}$, Carlo Dominici ${ }^{3}$, Ferdinando Romano ${ }^{4}$, Antonio Angeloni ${ }^{1}$, Cinzia Marchese ${ }^{1}$ and Simona Ceccarelli ${ }^{*^{*}}$ (I)

\begin{abstract}
Background: Ovarian cancer $(\mathrm{OC})$ is the most lethal gynecological malignancy and the second leading cause of cancer-related death in women. Treatment with PARP inhibitors (PARPi), such as Olaparib, has been recently introduced for OC patients, but resistance may occur and underlying mechanisms are still poorly understood. The aim of this study is to identify target genes within the tumor cells that might cause resistance to Olaparib. We focused on Neuropilin 1 (NRP1), a transmembrane receptor expressed in OC and correlated with poor survival, which has been also proposed as a key molecule in OC multidrug resistance.
\end{abstract}

Methods: Using three OC cell lines (UWB, UWB-BRCA and SKOV3) as model systems, we evaluated the biological and molecular effects of Olaparib on OC cell growth, cell cycle, DNA damage and apoptosis/autophagy induction, through MTT and colony forming assays, flow cytometry, immunofluorescence and Western blot analyses. We evaluated NRP1 expression in OC specimens and cell lines by Western blot and qRT-PCR, and used RNA interference to selectively inhibit NRP1. To identify miR-200c as a regulator of NRP1, we used miRNA target prediction algorithms and Pearsons' correlation analysis in biopsies from OC patients. Then, we used a stable transfection approach to overexpress miR-200c in Olaparib-resistant cells.

Results: We observed that NRP1 is expressed at high levels in resistant cells (SKOV3) and is upmodulated in partially sensitive cells (UWB-BRCA) upon prolonged Olaparib treatment, leading to poor drug response. Our results show that the selective inhibition of NRP1 is able to overcome Olaparib resistance in SKOV3 cells. Moreover, we demonstrated that miR-200c can target NRP1 in OC cells, causing its downmodulation, and that miR-200c overexpression is a valid approach to restore Olaparib sensitivity in OC resistant cells.

Conclusions: These data demonstrate that miR-200c significantly enhanced the anti-cancer efficacy of Olaparib in drug-resistant OC cells. Thus, the combination of Olaparib with miRNA-based therapy may represent a promising treatment for drug resistant $O C$, and our data may help in designing novel precision medicine trials for optimizing the clinical use of PARPi.

Keywords: Ovarian cancer, PARP inhibitors, NRP1, miRNAs, Drug resistance, miR-200c

\footnotetext{
* Correspondence: simona.ceccarelli@uniroma1.it

'Department of Experimental Medicine, Sapienza University of Rome, Viale

Regina Elena 324, 00161 Rome, Italy

Full list of author information is available at the end of the article
}

(C) The Author(s). 2019 Open Access This article is distributed under the terms of the Creative Commons Attribution 4.0 International License (http://creativecommons.org/licenses/by/4.0/), which permits unrestricted use, distribution, and reproduction in any medium, provided you give appropriate credit to the original author(s) and the source, provide a link to the Creative Commons license, and indicate if changes were made. The Creative Commons Public Domain Dedication waiver (http://creativecommons.org/publicdomain/zero/1.0/) applies to the data made available in this article, unless otherwise stated. 


\section{Background}

Ovarian cancer (OC) is the most lethal gynecological malignancy and the second leading cause of cancer-related death in women [1]. About $75 \%$ of patients are diagnosed at the late stage of the disease $[2,3]$, due to the lack of predictive biomarkers. The standard therapeutic protocol, including cisplatin-based combination chemotherapy and cytoreductive surgery, leads to an overall 5-year survival rate of only $15-30 \%$ for metastatic OC $[4,5]$, due to the onset of platinum resistance during the treatment [6]. In recent years, recognition of the role of inherited mutations in the DNA repair genes BRCA1 and BRCA2 in a proportion of OC patients led to the introduction of new therapeutic strategies targeting other DNA repair pathways, using poly-ADP ribose polymerase (PARP) inhibitors, such as Olaparib [7]. PARP inhibitors (PARPi) are able to abrogate PARP functionality, this bringing to the accumulation of single strand breaks (SSBs), which in turn are converted into double strand breaks (DSBs) that cells are not able to repair, causing cancer cell death [8]. PARPi act by blocking the catalytic domain of PARP enzymes, but these agents can also trap PARP proteins on the double-stranded DNA helix, this leading to cytotoxic lesions [9]. This strategy has been approved as treatment option for OC patients bearing BRCA1/2 gene mutation [10]. Moreover, it is now well established that a significant proportion of sporadic ovarian cancers present BRCA-like functional abnormalities (the so-called "BRCAness" syndrome), this opening the possibility of a wider application of treatment regimens specifically designed for familial BRCA-mutated tumors, such as PARPi $[11,12]$. However, long-term Olaparib administration might lead to PARPi resistance, which is currently under investigation. To date, the potential mechanisms involved in PARPi resistance are represented by DNA repair restoration [13, 14], PI3K/AKT pathway activation [15] and miRNA dysregulation [16-18], but further investigation is needed to clarify the complexities of pathways underlying PARPi-related clinical resistance.

Neuropilin 1 (NRP1) is a transmembrane glycoprotein belonging to a family of non-tyrosine kinase receptors [19-22], which acts as a receptor for various types of ligands, such as the class 3 semaphorins in neurons, the vascular endothelial growth factor (VEGF) family in endothelial cells, the plateletderived growth factor (PDGF) in megakaryocytes and the keratinocyte growth factor (KGF) in adipose-derived mesenchymal stem cells [23-25]. NRP1 has been shown to play a critical role in tumorigenesis, cancer invasion and angiogenesis, through the activation of VEGF, PI3K, and AKT pathways $[26,27]$. NRP1 protein is highly expressed in different cancer types, such as breast [28], colorectal [22], myeloid leukemia [29], glioma [20], pancreatic [30], and prostate [31] tumors. Some studies also reported an increased NRP1 expression in $\mathrm{OC}$ with respect to normal ovarian tissue [32] and to benign ovarian tumors [33]. In OC, NRP1 has been shown to promote unlimited growth through the evasion of contact inhibition [34], and higher NRP1 expression has been correlated with a shorter survival [32, 35], indicating that this protein could be a potential prognostic marker and a molecular target for therapy. Moreover, a comprehensive bioinformatics network analysis demonstrated that NRP1 is involved in multidrug resistance in OC [36].

miRNAs comprise a class of non-coding single-stranded RNAs containing about 21-24 nucleotides, encoded by endogenous genes, which can trigger target mRNA degradation or translation inhibition by targeting its 3 '-UTRs [37, 38]. miRNAs are involved in the post-transcriptional regulation of the expression of over $30 \%$ of human genes, thus impacting almost every cellular process, and changes in miRNAs expression play a key role in human pathologies, including cancer [39, 40]. In cancer, aberrantly expressed tumor-suppressor or oncogenic miRNAs are involved in tumor progression, metastasis, and drug resistance [41, 42]. So, the modulation of miRNA expression in cancer cells, through inhibiting oncogenic miRNAs or restoring tumorsuppressor miRNAs, could represent a viable approach for improving cancer therapy [43]. Some specific miRNAs, such as miR-148 and miR-124, have been shown to act as upstream suppressors of NRP1 signaling $[44,45]$. In cholangiocarcinoma, NRP1 contribution to the growth and metastasis of tumor cells is regulated by miR-320 [46]. In pancreatic cancer, NRP1 is negatively regulated by miR-141, a member of the miR-200 family, and the miR-141/NRP1 axis represents a potentially valuable diagnostic and therapeutic target for this tumor [47]. Almost all the members of miR-200 family (miR-200a, miR-200b, miR-200c and miR-141), have been shown to be upregulated in OC [48]. In particular, the function of miR-200c upregulation in OC $[49,50]$ is controversial, since it acts as a tumor-promoter by enhancing epithelial-mesenchymal transition, invasiveness, tumor growth and metastasis [51], but on the other hand its overexpression improves the response of $\mathrm{OC}$ to various chemotherapeutic agents [52], and its loss is associated with the acquisition of drug resistance [53]. Indeed, an interaction between miR-200 family and NRP1 3'UTR has been previously demonstrated by luciferase reporter experiments and Western blot assays in embryonic stem cells [54], but to date still little is known about the upstream miRNAs regulating NRP1 in OC and the molecular mechanisms by which miRNAs/NRP1 axis modulates drug resistance in this tumor. Therefore, the present study has been designed to investigate if miR-200c could regulate NRP1 in OC, and to assess how miRNA-regulated NRP1 contributes to PARPi resistance.

\section{Methods \\ OC tissue samples}

A total of 40 human ovarian specimens was obtained from patients who underwent surgical treatment at the 
Department of Maternal and Child and Urological Sciences at Sapienza University of Rome between November 2015 and July 2017. Ovarian tumor samples were obtained from n. 28 patients (median age 61 years; range 44-91 years) who underwent radical cytoreductive surgery followed by cisplatin-based chemotherapy $(\mathrm{CHT})$, while normal ovarian samples were obtained from n. 12 patients (median age 61 years; range 53-73 years) with benign ovarian cysts. The 28 patients diagnosed with OC included 26 patients with serous and 2 with mucinous carcinomas. Of the 28 carcinomas, 2 were classified as International Federation of Gynecology and Obstetrics (FIGO) stage II, 21 as stage III, and 5 as stage IV. With regard to the histologic grade, 5 were G1 and 23 were G3. In 16 cases, post-CHT samples were available for study. Patients provided their full consent for the donation of the tissue prior to any surgical procedure. All specimens were stored at $-80^{\circ} \mathrm{C}$ until RNA extraction.

\section{Cell cultures and treatments}

The human OC cell lines UWB1.289 (serous, BRCA1-null), UWB1.289 + BRCA1 (serous, BRCA1 restored), and SKOV3 were purchased from the American Type Culture Collection (ATCC-LGC Promochem, Teddington, UK). The UWB cell lines were cultured in a 1:1 mixture of RPMI-1640 (Sigma-Aldrich) and HUMEC (Thermo Fisher Scientific) medium, supplemented with $3 \%$ fetal bovine serum (FBS; Invitrogen) and antibiotics. The media for the UWB1.289 + BRCA1 cell line was further supplemented with $200 \mu \mathrm{g} / \mathrm{mL}$ of G-418 solution (Roche Diagnostics, Manheim, Germany) to maintain the expression of the BRCA1 protein. SKOV3 cells were maintained in RPMI (Sigma-Aldrich), supplemented with $10 \%$ FBS and antibiotics.

Olaparib (AZD-2281) was purchased from Selleckchem (Suffolk, UK) and used in vitro at concentrations ranging from 1.5 to $10 \mu \mathrm{M}$, for the indicated times. Olaparib powder was first dissolved at $10 \mathrm{mM}$ in dimethyl sulfoxide (DMSO; Sigma, St. Louis, MO, USA), diluted to its final concentration with culture medium, and freshly added to the cells every day for the duration of treatment. DMSO alone was used as control in untreated cells at $0.1 \%(\mathrm{v} / \mathrm{v})$ concentration. Chloroquine (CQ) was purchased from Sigma and used at a final concentration of $10 \mu \mathrm{M}$.

\section{Cell viability assay}

Cells were seeded onto 96-well plates at a density of $5 \times$ $10^{3}$ cells/well, then treated or not with Olaparib for 72 or $144 \mathrm{~h}$ at increasing concentrations $(1.5,5$ or $10 \mu \mathrm{M})$. At the end of time point, cells were incubated with $0.5 \%$ MTT (3-(4,5-dimethylthiazol-2-yl)-2,5-diphenyltetrazolium bromide; Sigma, St. Louis, MO, USA) for $4 \mathrm{~h}$ at $37^{\circ} \mathrm{C}$. The supernatant was then discarded, the MTT was dissolved with $100 \mu \mathrm{L}$ of DMSO and absorbance read at
$\mathrm{OD}=550 \mathrm{~nm}$ with an ELISA Microplate Reader (Bio-Rad, Hercules, CA, USA). Cell viability in Olaparib-treated cells was calculated in comparison to control samples (DMSO), arbitrarily set to $100 \%$, having six determinations per assay for each experimental condition.

\section{Colony formation assay}

Cells, previously treated with Olaparib for $144 \mathrm{~h}$, were seeded in 6-well plates in triplicate at a density of $2-4 \times$ $10^{3}$ cells/well, and incubated at $37^{\circ} \mathrm{C}$ for $10-14$ days to allow colonies to grow, with medium change every 3 days. Colonies were stained with $0.1 \%$ crystal violet for $5 \mathrm{~min}$ at room temperature (RT) and photographed. Then, crystal violet was solubilized in 30\% acetic acid in water for 15 min at RT, and absorbance was measured using the Biochrom Libra S22 UV/VIS spectrophotometer (Biochrom, Berlin, DE) at a wavelength of $595 \mathrm{~nm}$. 30\% acetic acid in water was used as blank control. Colony formation capacity in Olaparib-treated cells was calculated in comparison to control samples (DMSO), arbitrarily set to 1 .

\section{Immunofluorescence analysis}

Immunofluorescence was performed as previously described [55]. Cells, treated or not with Olaparib for 144 h, were seeded on coverslips onto 24-well plates at a density of $5 \times 10^{4}$ cells/well, and fixed in $4 \%$ paraformaldehyde for $30 \mathrm{~min}$ at room temperature, followed by treatment with $0.1 \mathrm{M}$ glycine in PBS for $20 \mathrm{~min}$ and with $0.1 \%$ Triton X-100 in PBS for additional 5 min to allow permeabilization. Cells were then incubated with antiphospho-histone H2A.X (Ser139) antibodies ( $\gamma \mathrm{H} 2 \mathrm{AX}$; Cell Signalling, Inc. Danvers, MA, USA). After appropriate washing in PBS, primary antibodies were visualized using TexasRed-conjugated goat anti-rabbit IgG (Jackson ImmunoResearch Laboratories, West Grove, PA, USA). Nonspecific fluorescence was determined by omitting primary antibody. Nuclei were visualized using 4',6-diamidino-2-phenylindole dihydrochloride (DAPI) (SigmaAldrich). The single stained and merged images were acquired with a Zeiss ApoTome microscope (40× magnification) using the Axiovision software (Carl Zeiss, Jena, Germany). $\gamma \mathrm{H} 2 \mathrm{AX}$ fluorescence intensity was measured using ImageJ software (v. 10.2), evaluating at least six random microscopic fields for each condition.

\section{Cell cycle and apoptosis analysis by flow cytometry}

For cell cycle analysis, cells were treated with $1.5 \mu \mathrm{M}$ and $5 \mu \mathrm{M}$ Olaparib for $72 \mathrm{~h}$, then collected and washed twice with phosphate buffered saline (PBS). After fixation in $70 \%$ ice-cold ethanol overnight at $4{ }^{\circ} \mathrm{C}$, cell pellets were washed twice with ice-cold PBS and treated with RNase A for $15 \mathrm{~min}$ at $37^{\circ} \mathrm{C}$. Propidium iodide (PI) was added to each sample and DNA content was determined by collecting 10,000 events using a BD FACS 
Calibur Flow Cytometer (BD Biosciences). Data were analyzed using ModFit 3.1 software (BD Biosciences).

Apoptosis was analyzed by using Annexin A5 FITC/7AAD Kit (Beckman Coulter), following the manufacturer's instructions. Briefly, cells were treated with Olaparib for 144h. Approximately $2 \times 10^{5}$ cells were stained with Annexin A5 FITC and 7-Amino-Actinomycin (7-AAD) for $15 \mathrm{~min}$ at RT in the dark. Fluorescence intensities of treated samples and controls were collected with a CytoFLEX flow cytometer (Beckman Coulter, Germany). Quadrant analysis was performed using the Kaluza software (Beckman Coulter) to quantify viable cells (7-AAD-negative/Annexin A5-negative), early apoptotic cells (Annexin A5-positive/7-AAD-negative), and late apoptotic cells (Annexin A5-positive/7-AAD-positive). Experiments were performed at least twice. For each point, the sum of early and late apoptotic cells was plotted.

\section{Western blot analysis}

Cells, treated or not with $1.5 \mu \mathrm{M}$ and $5 \mu \mathrm{M}$ Olaparib for the indicated times, were lysed in RIPA buffer. Total proteins $(50-100 \mu \mathrm{g})$ were resolved under reducing conditions by 7-15\% SDS-PAGE and transferred to Immobilon-FL membranes (Merck Millipore, Billerica, MA, USA), as previously described [56]. Membranes were blocked in TBS containing $0.1 \%$ Tween 20 (TBS-T) and $5 \%$ milk for $1 \mathrm{~h}$ at $25^{\circ} \mathrm{C}$ and then incubated overnight at $4{ }^{\circ} \mathrm{C}$ with the following primary antibodies: anti-Neuropilin 1 (A-12) (NRP1) (Santa Cruz Biotechnology, Santa Cruz, CA, USA), anti-phosphohistone H2A.X (Ser139) ( $\mathrm{H}$ 2AX), anti-Cyclin B1, antiphospho-AKT (Ser473) (phAKT), anti-AKT, anti-cleaved Caspase-3, anti-cleaved PARP1 (PARP1) (Cell Signalling) and anti- $\beta$-Tubulin (Sigma-Aldrich). Membranes were then incubated with the appropriate horseradish peroxidase(HRP-) conjugated secondary antibody (Santa Cruz Biotechnology) for $1 \mathrm{~h}$ at $25^{\circ} \mathrm{C}$. Bound antibody was detected by enhanced chemiluminescence detection reagents (Pierce Biotechnology Inc., Rockford, IL, USA), according to the manufacturer's instructions. Tubulin served to estimate the protein equal loading. Densitometric analysis was performed using Quantity One Program (Bio-Rad Laboratories S.r.l., Segrate, MI, Italy).

\section{Quantitative real-time PCR (qRT-PCR)}

Cells were harvested and total RNA was extracted with the use of TRIzol reagent (Invitrogen). Quantity and quality of the extracted RNA were assessed by NanoDrop (Thermo Fisher Scientific). For mRNA detection, cDNA was generated with oligo (dT) from $1 \mu \mathrm{g}$ of RNA using the SuperScript III Reverse Transcriptase Kit (Invitrogen). Quantitative real-time PCR assays (qRT-PCR) were conducted in triplicate on an ABI 7500 Real Time instrument (Applied Biosystems by Life Technologies, Carlsbad, CA, USA) as previously described [57]. Briefly, the abundance of NRP1 was quantified using the appropriate Taq-Man gene expression assay kit (Applied Biosystems). $\beta$-actin mRNA was used as endogenous control.

For miRNA detection, $40 \mathrm{ng}$ of RNA were retrotranscribed with a specific primer for miR-200c-3p (Thermo Fisher Scientific). Expression of miR-200c-3p was analyzed by using sequence-specific TaqMan MicroRNA Assays (Applied Biosystems). U6 small nuclear RNA levels were used as internal control.

\section{siRNA-mediated downregulation of NRP1}

The NRP1-specific (siNRP) short interfering RNA, which specifically knock down NRP1 gene expression, as well as negative control siRNA (siNC), which does not lead to the specific degradation of any cellular mRNA, was purchased from Santa Cruz Biotechnology. SKOV3 cells were seeded in 6 well-plate at a density of $0.8 \times 10^{5}$ cells/well and transfected with siRNA at a final concentration of $50 \mathrm{nM}$ using the HiPerfect Transfection Reagent (Qiagen, Valencia, CA, USA) according to the manufacturer's instructions for long term transfection. The achievement of an efficient knockdown without cytotoxicity at $144 \mathrm{~h}$ after the initial transfection was confirmed by performing a timecourse experiment. Silenced cells, treated or not with Olaparib $(5 \mu \mathrm{M})$, were collected and processed for RNA and protein extraction. NRP1 silencing was confirmed by both Western blot and qRT-PCR experiments.

miRNA conserved target sites prediction in 3' UTR of NRP1

The RNA22 v2 (https://cm.jefferson.edu/rna22/Interactive/ ) and the TargetScan (http://www.targetscan.org/vert_72/) predictions were used to identify the putative miRNA target sites in 3' UTRs of NRP1 gene. The NRP1 gene symbol and human species were retrieved from the database. The 3' UTR of NRP1 transcript ENST00000374875.1 was selected to analyze the potential binding site of miRNAs.

\section{Transfection of miR-200c in SKOV3 cell line}

Plasmid vector encoding miR-200c and empty pCMV vector were obtained from OriGene Company. Both vectors had Geneticin (G418) resistance as a marker for screening aims. SKOV3 cells were seeded in a 12 well-plate at a density of $0.5 \times 10^{6}$ cells/well and transfected with $1 \mu \mathrm{g}$ of pCMV-miR-200c plasmid (miR-200c) or the corresponding empty vector (CTRL) using Lipofectamine 3000 (ThermoFisher Scientific), following the manufacturer's instructions. $48 \mathrm{~h}$ post-transfection, cells were resuspended in fresh culture medium supplemented with 0.5 $\mathrm{mg} / \mathrm{ml} \mathrm{G418}$ and distributed in 96 well-plate. The cells were kept under G418 selection for a couple of weeks in order to obtain G418 resistant clones. One clone from each transfection with pCMV empty vector and pCMVmiR-200c was obtained and used in our studies. 


\section{Statistical analysis}

All data reported were verified in at least two different experiments and plotted as means \pm standard deviations. The differences between control and experimental groups were analyzed by GraphPad Prism 7, using twotailed unpaired $t$ test. Pearson's coefficient correlation was used for correlation assay. $P$ values $<0.05$ were considered as statistically significant.

\section{Results}

Variable cytotoxic effects of prolonged Olaparib treatment in different OC cell lines are mediated by differential DNA damage repair and activation of apoptosis/autophagy.

We first confirmed the differential effect of Olaparib treatment on OC cell lines depending on BRCA status, by performing a dose- and time-curve evaluation of cell viability through MTT assay in the BRCA1-null UWB1.289 cell line (UWB), the UWB1.289 + BRCA1 cells (UWBBRCA), in which BRCA1 expression was permanently restored, and the BRCA wild-type SKOV3 cell line. As expected, the sensitivity of the BRCA1-null UWB cells to Olaparib was greater than both its BRCA1 restored counterpart UWB-BRCA and the BRCA wild-type SKOV3 cells (Additional file 1: Figure S1). Olaparib, by inhibiting PARP proteins, rapidly induces DNA damage, which can be measured by $\gamma \mathrm{H} 2 \mathrm{AX}$ expression at $24 \mathrm{~h}$, in the three cell lines. In particular, evaluation of $\gamma \mathrm{H} 2 \mathrm{AX}$ foci by both immunofluorescence (IF) and Western blot analysis after prolonged Olaparib treatment $(144 \mathrm{~h})$ confirmed the persistence of DNA damage only in cells with impaired DNA repair (UWB cells) (Additional file 1: Figure S2). Cell cycle analysis of the three cell lines showed a significant arrest in G2 phase (4n) upon Olaparib treatment, with a corresponding decrease of cell percentage in both G1 (2n) and $S$ phases, particularly evident in UWB and UWB-BRCA cells. Consistent with this observation, cells exposed to Olaparib and, particularly, UWB and UWB-BRCA cells, showed increased expression of Cyclin B1, a G2/M-regulating protein. The distribution of cell cycle fractions in G1, S and G2 phase and the expression of Cyclin B1 are shown in Additional file 1: Figure S3.

When assessing the effect of prolonged Olaparib treatment $(144 \mathrm{~h})$ on colony forming efficiency, we observed that Olaparib significantly inhibited clonogenic ability of BRCA-null UWB cells, while it had no effect on the BRCA wild-type SKOV3 cells, as expected (Fig. 1). However, interestingly, a partial inhibition was achieved at the higher Olaparib dose in UWB-BRCA cells (60\% inhibition compared to control samples; Fig. 1).

These data confirmed what previously observed about Olaparib effects [7] but also pointed out a partial efficacy of prolonged Olaparib treatment on UWB-BRCA cells. So, we investigated if the variable response of UWB-BRCA cells upon Olaparib treatment was due to the induction of different intracellular pathways. We assessed the induction of apoptosis by performing flow cytometry assays with Annexin A5 FITC/7-AAD double staining. Treatment of UWB-BRCA and SKOV3 cell lines with Olaparib $(5 \mu \mathrm{M})$ for $144 \mathrm{~h}$ did not significantly increase the percentage of cells undergoing early or late apoptosis when compared to mocked control cells. Indeed, Olaparib treatment induced a consistent increase in the number of apoptotic UWB cells (from about $10 \%$ in DMSO to almost $21 \%$ in $5 \mu \mathrm{M}$ Olaparib) (Fig. 2a). So, Annexin A5 FITC/7-AAD double staining confirmed that cytotoxic effects of Olaparib were evident only in UWB cells. Furthermore, Western blot analysis showed that both caspase- 3 and PARP1 cleavage/activation were strongly evident only in UWB cells upon Olaparib treatment at both doses (Fig. 2b), in accordance with the data obtained by FACS analysis.

Since PI3K/AKT molecular pathway is a pivotal signal involved in cell survival and apoptosis, we also assessed by Western blot analysis the activation of AKT phosphorylation at Ser473. As reported in Fig. 2b, phosphorylation levels of AKT protein (phAKT) upon treatment with Olaparib were unaffected in SKOV3 cells, and markedly reduced in a dose-dependent way in UWB cells, whilst AKT total levels were unchanged, this confirming the central role of the AKT signal transduction pathway in the Olaparib-mediated cell death. Interestingly, the treatment of UWB-BRCA cells with Olaparib led to a significant downregulation of phAKT expression, without concomitant upregulation of cleaved Caspase 3 and cleaved PARP1 expression.

Since AKT represents a key inhibitor of autophagy, we explored if the reduced phosphorylation state of AKT in UWB-BRCA cells was suggestive of autophagy activation. The classical hallmarks of autophagy, represented by LC3-I to LC3-II conversion and P62 protein degradation, were evaluated through Western blot analysis (Fig. 2c). We observed a significant increase in the LC3-II/ LC3-I ratio in UWB-BRCA cells treated with Olaparib 1.5 or $5 \mu \mathrm{M}$ (2.3 and 2.5 fold, respectively). Autophagy activation in these cells was also confirmed by the degradation of $\mathrm{P} 62$ protein (0.7 and 0.3 fold, respectively). Conversely, UWB and SKOV3 cells did not show a significant modulation of both LC3-II/LC3-I ratio and P62 (Fig. 2c). To investigate the involvement of autophagy in PARPi-resistance, we assessed apoptosis induction upon Olaparib treatment in the presence or not of the autophagy inhibitor Chloroquine (CQ). Treatment with CQ induced the accumulation of LC3-II and P62 in both UWB and UWB-BRCA cell lines (Fig. 2d), this reflecting autophagy inhibition. Concurrent treatment with Olaparib and CQ did not induce apoptosis activation in UWB-BRCA cells and significantly decreased the expression of the apoptosis markers cleaved Caspase 3 and cleaved PARP1 in UWB cells (Fig. 2d). These results 


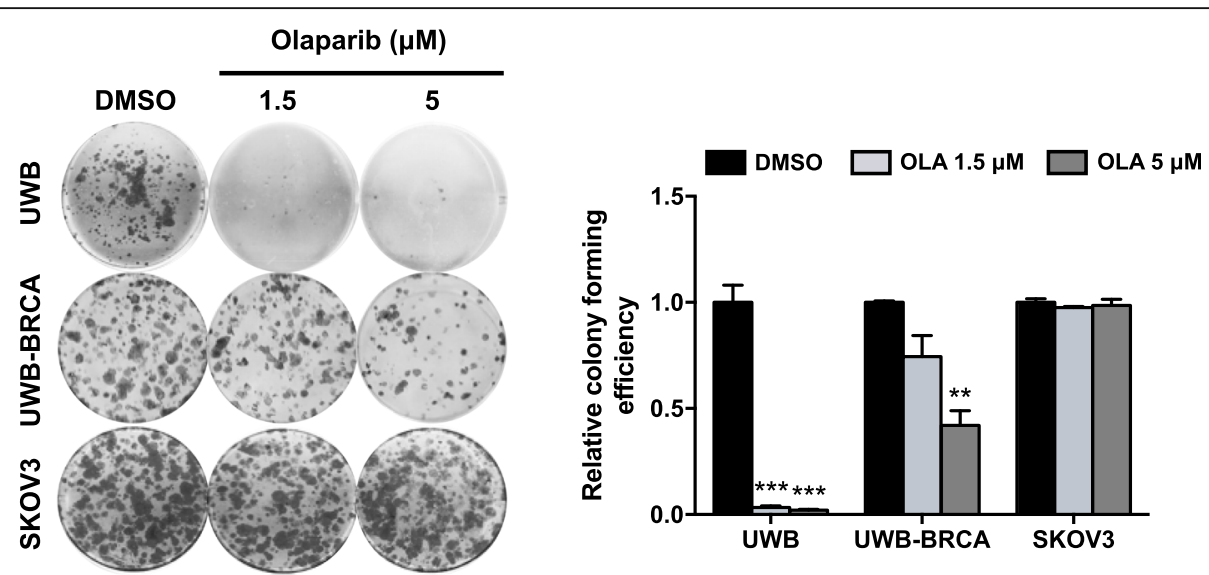

Fig. 1 Differential effect of Olaparib on clonogenic ability of OC cell lines. UWB, UWB-BRCA and SKOV3 cells were treated with Olaparib for $144 \mathrm{~h}$, then seeded at low concentration and allowed to grow for 12 days. The effect of Olaparib on cell clonogenicity was determined by colony formation assay. The images are representative pictures of colonies stained with crystal violet. Colony forming efficiency was calculated by crystal violet absorbance. Mean values obtained from two independent experiments, each performed in triplicate, are reported in graph. Error bars represent standard deviations. ${ }^{* *}, p<0.005,{ }^{* * *}, p<0.0005$ vs. control (DMSO)

suggest that in OC cell lines autophagy does not represent a cytoprotective response, but rather contributes to the cytotoxic effect of Olaparib.

\section{Olaparib treatment modulates NRP1 expression in UWB and UWB-BRCA cells}

NRP1 is a membrane receptor known to promote tumor growth and drug resistance in several cancers, including OC $[19,30,34,36]$. Firstly, we performed qRT-PCR to assess the mRNA expression of NRP1 in ovarian cancer specimens. In contrast with previous observations [32], we found that NRP1 expression was significantly lower in OC samples ( 0.6 fold change with respect to normal ovarian tissue) (Fig. 3a). A low NRP1 protein expression was also confirmed in UWB and UWB-BRCA cells, derived from serous $\mathrm{OC}$, whilst SKOV3 cells, derived from the ascites of an ovarian adenocarcinoma patient, showed a high basal expression of NRP1 protein (5 fold, with respect to UWB cells) (Fig. 3b). This differential expression among the three cell lines was also confirmed at mRNA level by qRT-PCR (Fig. 3c). We then investigated if chemotherapeutic treatment would be able to change NRP1 expression in OC patients. A total of 16 OC samples from the patient cohort were selected, based on the availability of pre- and post-CHT samples, and NRP1 expression was assessed in CHT-naive and post-CHT OC samples by qRT-PCR. We identified a significantly increased expression of NRP1 mRNA transcript in post-CHT samples (1.7 fold increase with respect to pre-CHT samples) (Fig. 3d). Such correlation between $\mathrm{CHT}$ and increase of NRP1 expression supports the hypothesis of a role of NRP1 in drug response and potentially drug resistance. To test this hypothesis in our model, we assessed NRP1 expression in OC cell lines after treatment with Olaparib $(1.5$ and $5 \mu \mathrm{M})$ for $144 \mathrm{~h}$. As shown in Fig. 3e, the treatment induced an upregulation of NRP1 protein in UWB and UWB-BRCA cell lines at both doses (1.7 and 3.1 fold in UWB cells and 1.8 and 7.3 fold in UWB-BRCA cells, respectively). Such upmodulation was confirmed to be significant at mRNA level only in UWB-BRCA cells (Fig. 3f). As for SKOV3 cells, Olaparib treatment did not affect NRP1 protein or mRNA expression (Fig. 3e, f). Our results suggest that Olaparib-mediated upregulation of NRP1 in OC cell lines expressing low basal levels of this protein might represent a drug resistance mechanism.

\section{NRP1 silencing restores sensitivity to Olaparib treatment in resistant $O C$ cells}

To confirm the role of NRP1 in Olaparib resistance, we introduced a specific siRNA (siNRP) in the drug-resistant SKOV3 cell line. The efficiency of NRP1 silencing was assessed at 48, 72 and $144 \mathrm{~h}$ by Western blot analysis. As reported in Fig. 4a, a significant reduction of NRP1 expression (70\%) was achieved as early as $48 \mathrm{~h}$ after silencing ( 0.3 fold with respect to nonspecific control siRNA, siNC), then reached $90 \%$ efficiency after $144 \mathrm{~h}$ (0.1 fold). NRP1 silencing was also performed on SKOV3 cells untreated or treated with Olaparib $(5 \mu \mathrm{M})$ for $144 \mathrm{~h}$. Western blot analysis confirmed that Olaparib treatment did not affect NRP1 silencing efficiency ( 0.03 fold versus siNC in both untreated and treated cells) (Fig. 4b). Then, cells were subjected to both MTT and clonogenic assay, to assess the effect of NRP1 silencing on SKOV3 response to Olaparib. The MTT assay demonstrated that viability of NRP1silenced cells was reduced by $36 \%$ upon Olaparib treatment, whilst siNC-transfected cells treated with Olaparib showed a $7 \%$ reduction with respect to DMSO controls 


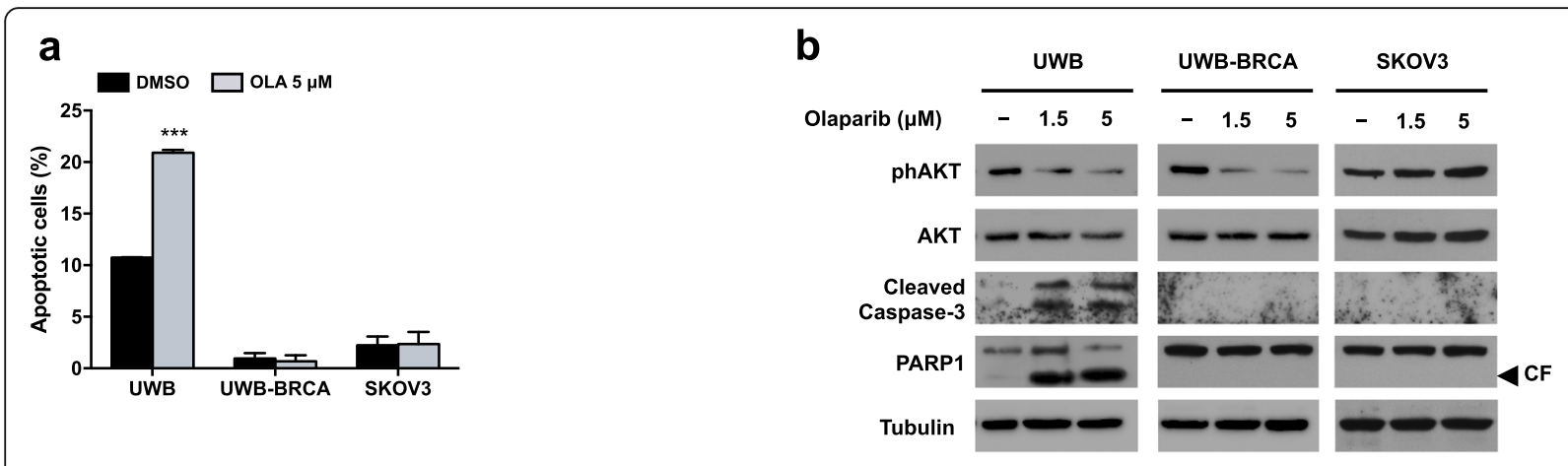

C
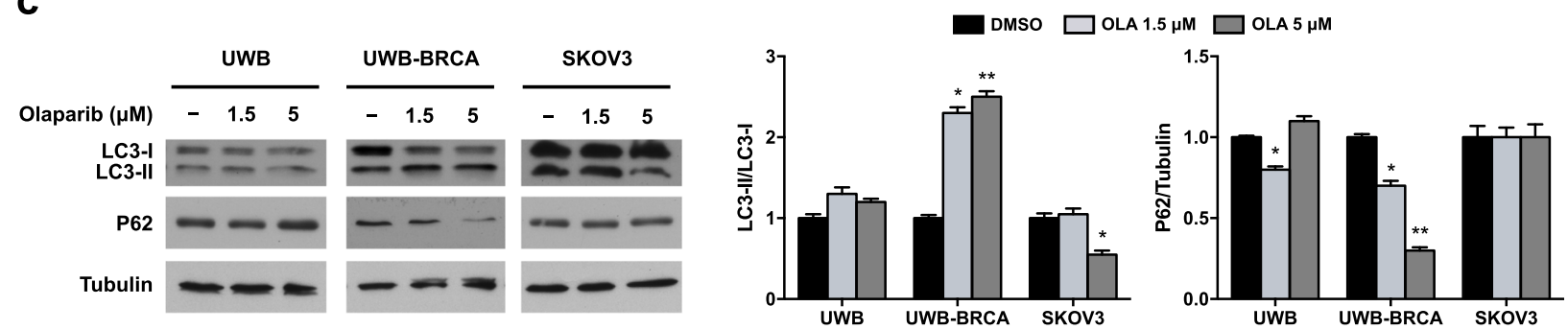

d
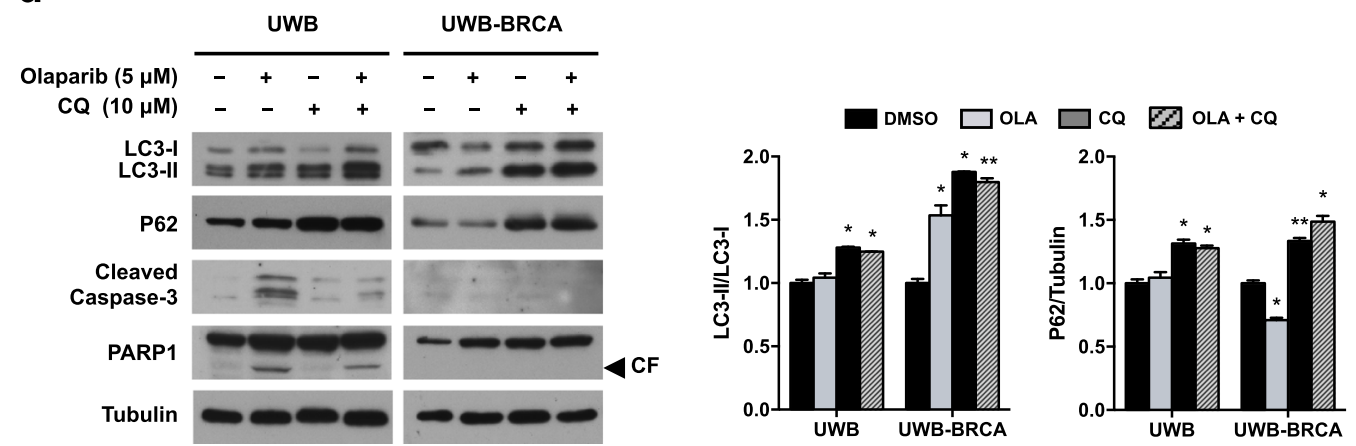

Fig. 2 Effects of Olaparib treatment on apoptosis/autophagy induction in OC cell lines. UWB, UWB-BRCA and SKOV3 cells were treated for $144 \mathrm{~h}$ with Olaparib. a) The percentages of early apoptotic and late apoptotic cells were obtained by flow cytometry quadrant analysis with annexin A5 FITC/7-AAD double staining, and expressed as histograms. b) The expression of the apoptosis related proteins phAKT (Ser473), AKT, cleaved Caspase 3 and cleaved PARP1 was determined by Western blot analysis. Tubulin expression was used as internal control. The images are representative of at least two independent experiments. CF, cleaved form of PARP1. c) The expression of the autophagy related proteins LC3 and P62 was assessed by Western blot analysis. d) UWB and UWB-BRCA cells were treated for $144 \mathrm{~h}$ with Olaparib (5 $\mu \mathrm{M}), \mathrm{Chloroquine}(\mathrm{CQ}, 10 \mu \mathrm{M})$ or a combination of them, and the expression of LC3 and P62 was assessed. The intensity of the LC3-II and P62 bands was evaluated by densitometric analysis, normalized with LC3-I and Tubulin, respectively, and reported in graph. Error bars represent standard deviations. * $p<0.05$, **, $p<0.005$ vs. control (DMSO)

(Fig. 4c). As shown in Fig. 4d, Olaparib treatment did not affect clonogenicity of siNC cells, whilst in NRP1-silenced cells it determined a significant reduction in colony formation capacity ( 0.2 fold with respect to DMSO controls). So, NRP1 silencing was able to restore Olaparib efficacy. Moreover, the role of NRP1 in PARPi resistance is further confirmed by Western blot analysis assessing apoptosis pathway activation (reduction of AKT phosphorylation, cleavage of Caspase 3 and PARP1) in NRP1-silenced SKOV3 cells treated with Olaparib (Fig. 4e). Altogether, these data demonstrate that: i) inhibition of NRP1 restored the sensitivity of drug-resistant cells to Olaparib, ii) NRP1 signaling axis is an important determinant of PARPi tolerance, and iii) modulation of NRP1 expression represents a potential approach to overcome drug resistance in OC.

NRP1 expression in OC cell lines is regulated by miR-200C As demonstrated above, high expression of NRP1 is correlated with PARPi-resistance of SKOV3 cells. We employed two widely used miRNA target prediction algorithms, 
a

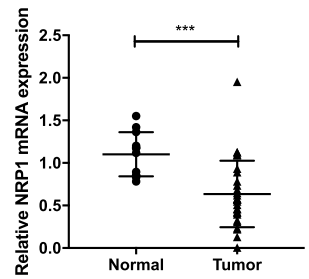

d

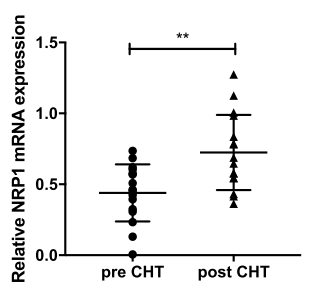

b

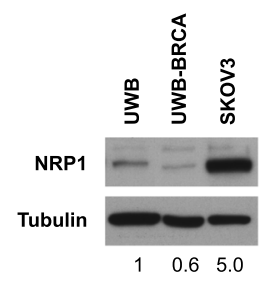

C

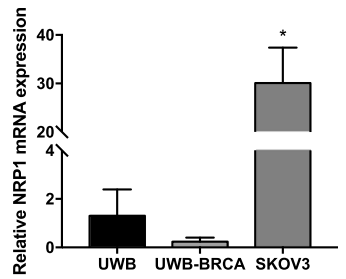

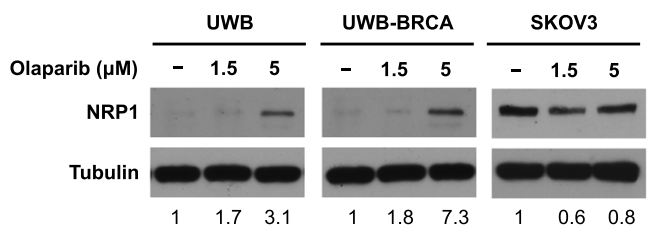

$\mathbf{f}$

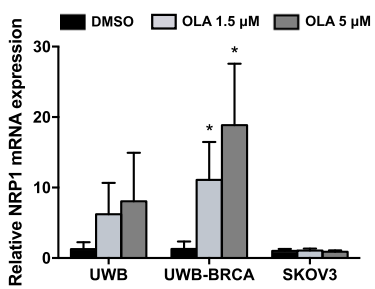

Fig. 3 Effect of Olaparib on NRP1 expression in OC. a) The expression pattern of NRP1 was assessed by qRT-PCR assays in OC tumor tissues from 28 patients and noncancerous ovarian tissues from 12 healthy donors. ${ }^{* *}, p<0.0005$. b, c) Basal NRP1 protein (b) and mRNA (c) levels in UWB, UWB-BRCA and SKOV3 cells were assessed by Western blot analysis and qRT-PCR, respectively. d) The expression pattern of NRP1 was assessed by qRT-PCR assays in OC tumor biopsies from 16 patients obtained before and after platinum-based chemotherapy (CHT). **, $p<0.005$. e, f) NRP1 protein $(\mathbf{e})$ and mRNA (f) expression after Olaparib treatment for $144 \mathrm{~h}$ were evaluated by Western blot analysis and qRT-PCR, respectively. For Western blot analysis, tubulin expression was used as internal control, and the images are representative of at least three independent experiments. The intensity of the bands was evaluated by densitometric analysis, normalized and reported as relative expression with respect to control (DMSO). For qRT-PCR, each experiment was performed in triplicate and mRNA levels were normalized to GAPDH mRNA expression. Error bars represent standard deviations. *, $p<0.05$ vs. UWB cells (c) or vs control (DMSO) (f)

RNA22 v2 (https://cm.jefferson.edu/rna22/Interactive/) and TargetScan Human 7.2 (http://www.targetscan.org/vert_72/ ), to pull potential miRNAs that directly regulate NRP1. In particular, we found two conserved binding sites for the miR-200 family members (one for miR-141-3p/200a-3p and another for miR-429/200bc-3p), which have been previously demonstrated to directly target the 3'UTR of the most prevalent NRP1 transcript [54] (Fig. 5a). Indeed, we focused on miR-200c-3p (from now on referred to as miR200c), which is predicted to target NRP1 with a percentile score of $68 \%$ with 7-nucleotide complementarity (Fig. 5a), since it has been previously identified as an inducer of sensitivity against various anti-cancer agents [52], and its low expression has been implicated in paclitaxel resistance in OC [51]. We observed that the expression level of miR200c was higher in OC specimens with respect to normal ovarian tissue (Fig. 5b), in accordance to what previously reported [48], and is negatively correlated with that of NRP1 in OC specimens, as shown by correlation analysis
(Fig. 5c). Such inverse correlation was also confirmed at protein and mRNA levels in OC cells. In fact, miR-200c basal expression was consistently high in UWB-BRCA cells (5 fold, with respect to UWB cells) and very low in SKOV3 cells ( 0.05 fold, with respect to UWB cells) (Fig. $5 d$ ). The increase of NRP1 expression upon treatment in UWB and UWB-BRCA corresponded to a significant decrease of miR-200c expression in these cells, with no variations in SKOV3 cells, in which Olaparib did not modulate NRP1 levels (Fig. 5e). These data supported the role of miR-200c as a negative regulator of NRP1 in OC.

To further validate our hypothesis of NRP1/miR-200c interaction, we investigated the effects of miR-200c overexpression in SKOV3 cells, expressing low levels of miR200c and high levels of NRP1. First, we confirmed that transfection of miR-200c mimics into SKOV3 cells significantly downmodulated NRP1 expression, as reported in Additional file 1: Figure S4. Cells were then stably transfected with a plasmid carrying the precursor of miR-200c 


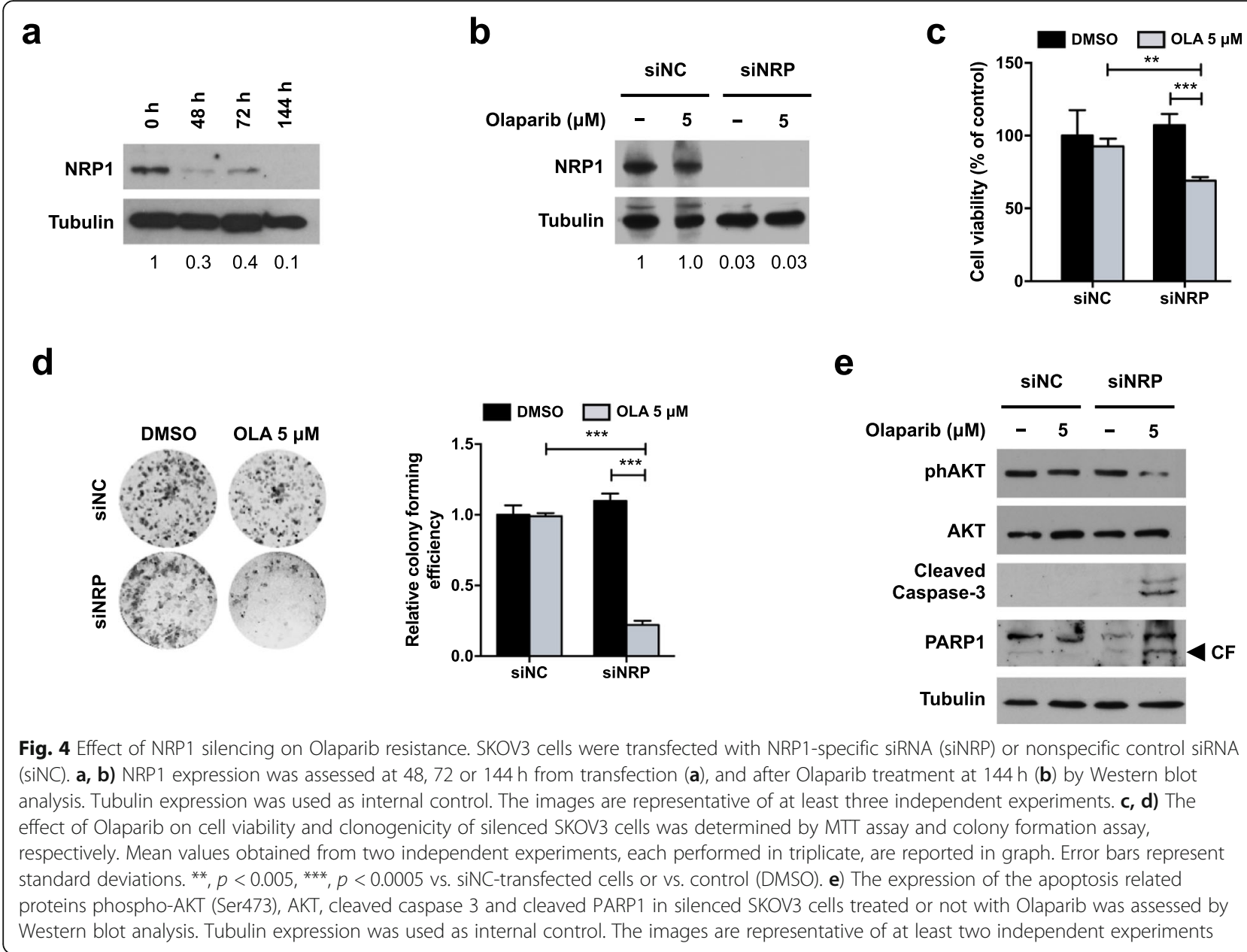

(pCMV-miR-200c) and its corresponding vector control (pCMV), and the expression levels of miR-200c were measured by qRT-PCR. We observed that miR-200c was highly increased in the pCMV-miR-200c group (miR200c) compared to pCMV empty vector (CTRL) (Fig. 6a), this confirming the miRNA transfection efficiency. Both mRNA and protein expression of NRP1 were assessed in miR-200c-transfected SKOV3 cells by qRT-PCR and Western blot analysis, respectively. Exogenous expression of miR-200c could effectively decrease the levels of NRP1 protein by $80 \%$ (Fig. $6 \mathrm{~b}$ ) and of NRP1 mRNA by 60\% (Fig. 6c).

\section{miR-200c sensitizes resistant SKOV3 cells to Olaparib by activating apoptosis}

To confirm the role of miR-200c in Olaparib resistance, SKOV3 cells stably transfected with pCMV-miR-200c (miR-200c) or empty pCMV vector (CTRL) were treated or not with Olaparib $(5 \mu \mathrm{M})$ for $144 \mathrm{~h}$ and used for functional assays. The results of MTT assay revealed that, in miR-200c-transfected SKOV3 cells, Olaparib treatment reduced viability by about $37 \%$, compared to the untreated samples, whilst the reduction of viability after Olaparib treatment was only $9 \%$, compared to DMSO controls, in cells transfected with CTRL vector (Fig. 7a). Similarly, a significant decrease in colony forming efficiency after Olaparib treatment was observed only in miR-200c-transfected cells (0.6 fold with respect to untreated controls) (Fig. 7b). We investigated if miR-200c overexpression would influence SKOV3 ability to repair DNA damage induced by Olaparib. To test this hypothesis, we performed immunofluorescence staining to measure the intensity of $\gamma \mathrm{H} 2 \mathrm{AX}$ foci in miR-200ctransfected and CTRL-transfected SKOV3 cells after Olaparib treatment at $144 \mathrm{~h}$. As described above for parental SKOV3 cells, at $144 \mathrm{~h} \gamma \mathrm{H} 2 \mathrm{AX}$ expression levels in CTRL-transfected cells were similar between Olaparib-treated cells and DMSO controls, while we observed an increase of $\gamma \mathrm{H} 2 \mathrm{AX}$ expression after Olaparib treatment in miR-200c-transfected cells (Fig. 7c). Such results suggest that miR-200c overexpression might hinder DNA repair in Olaparib-resistant cells, this leading to a persistent DNA damage and a subsequently greater effectiveness of PARPi. 


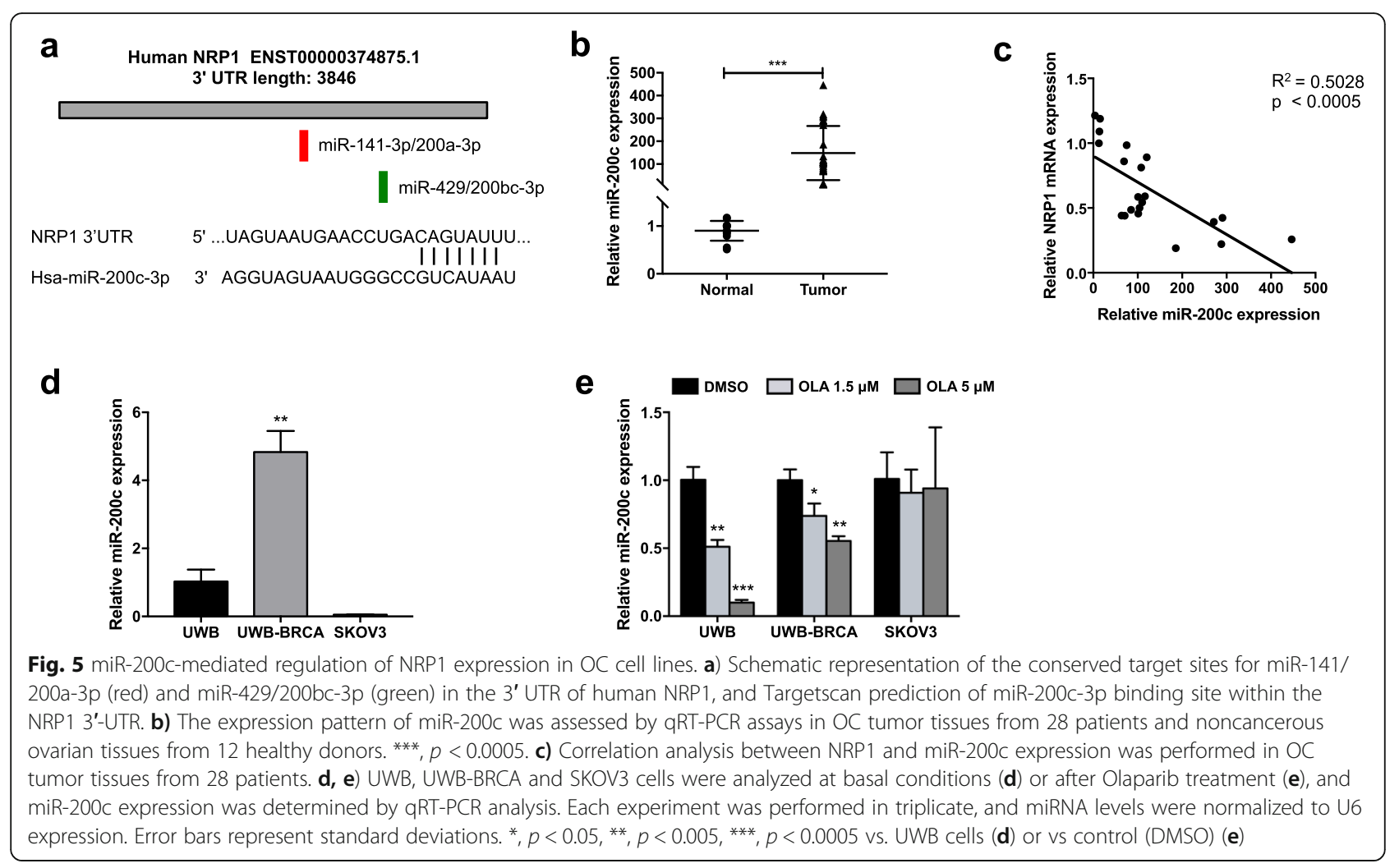

To confirm such conclusion, we assessed if miR-200c overexpression was able to restore PARPi ability to induce apoptosis. Flow cytometry assays with Annexin A5 FITC/ 7-AAD double staining demonstrated that Olaparib treatment significantly increased the percentage of apoptotic cells in SKOV3 overexpressing miR-200c (from around $2 \%$ in DMSO to approximately $10 \%$ in $5 \mu \mathrm{M}$ Olaparib) and not in CTRL-transfected cells (Fig. 8a). Furthermore, Western blot analysis showed expression of cleaved Caspase 3 and cleaved PARP1 upon Olaparib treatment only in the miR-200c-transfected cells, and not in the CTRLtransfected cells (Fig. 8b). These data strongly suggested that miR-200c regulates apoptosis induction by Olaparib in SKOV3 cells. In agreement with what observed for UWB cells, Olaparib treatment at $144 \mathrm{~h}$ did not induce autophagy activation in miR-200c-transfected cells, as reported in Additional file 1: Figure S5.

Altogether, our data suggest that miR-200c overexpression is able to restore SKOV3 susceptibility to Olaparib by targeting NRP1. a

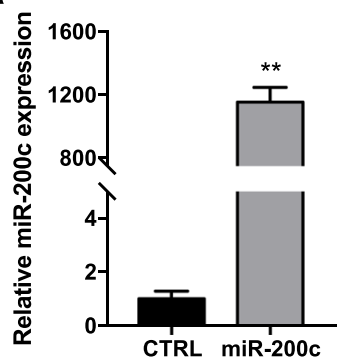

b

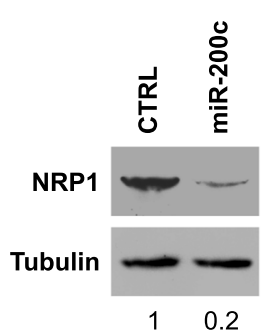

C

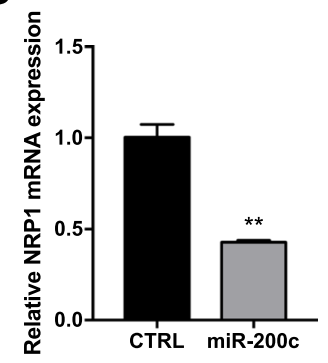

Fig. 6 Effect of miR-200c overexpression on NRP1. SKOV3 cells were stably transfected with a plasmid carrying the precursor of miR-200c (miR200c) and its corresponding vector control (CTRL). miR-200c expression was assessed by qRT-PCR analysis (a). NRP1 protein (b) and mRNA (c) expression were evaluated by Western blot analysis and qRT-PCR, respectively. For Western blot analysis, tubulin expression was used as internal control, and the images are representative of at least three independent experiments. The intensity of the bands was evaluated by densitometric analysis, normalized and reported as relative expression with respect to control (CTRL). For qRT-PCR, each experiment was performed in triplicate. miRNA levels were normalized to U6 expression, while mRNA levels were normalized to GAPDH mRNA expression. Error bars represent standard deviations. ${ }^{* *}, p<0.005$ vs. CTRL 
a

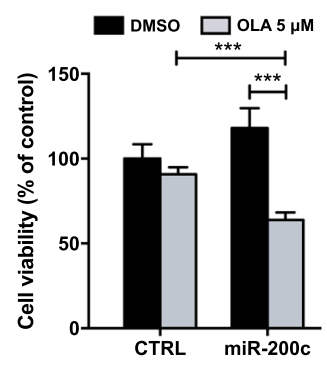

C

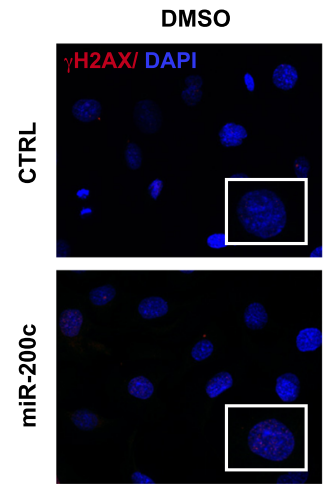

b

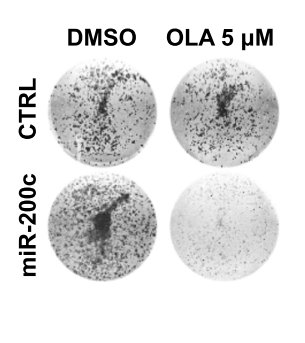

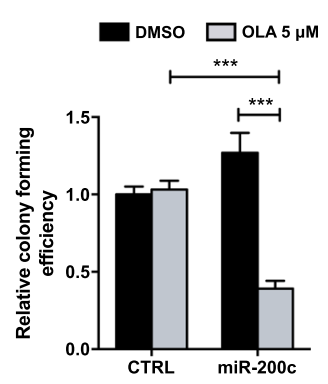

OLA $5 \mu \mathrm{M}$
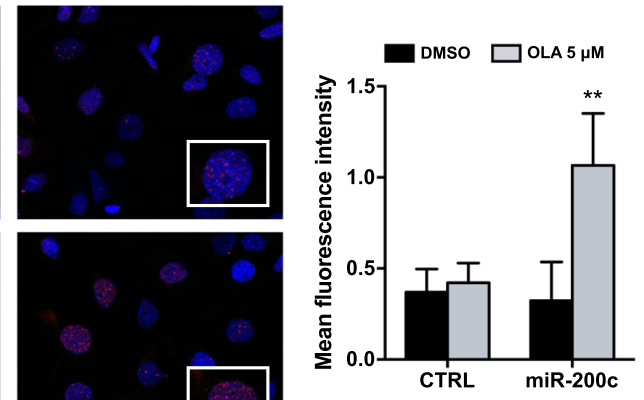

Fig. 7 Effect of miR-200c overexpression on SKOV3 response to Olaparib. SKOV3 cells were stably transfected with a plasmid carrying the precursor of miR-200c (miR-200c) and its corresponding vector control (CTRL), then treated for $144 \mathrm{~h}$ with Olaparib. a, b) Cell viability and colony forming efficiency were determined by MTT assay and clonogenic assay, respectively. Mean values obtained from two independent experiments, each performed in triplicate, are reported in graph. Error bars represent standard deviations. ${ }^{* *}, p<0.0005$ vs. CTRL cells or vs. control (DMSO). c) The presence of $\mathrm{YH} 2 \mathrm{AX}$ foci (red) was assessed by immunofluorescence analysis. Nuclei (blue) were visualized with 4', 6-diamidino-2-phenylindole (DAPI). Images were captured under ApoTome microscope at 40x magnification. Quantification of $\mathrm{YH} 2 \mathrm{AX}$ foci was determined by measuring red fluorescence intensity with ImageJ software. Mean values obtained from measurements of five microscopic fields randomly taken from three independent experiments are reported in graph. Error bars represent standard deviations. ${ }^{*}, p<0.005$ vs. control (DMSO)

a

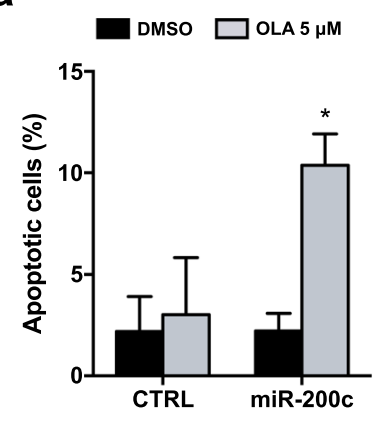

b

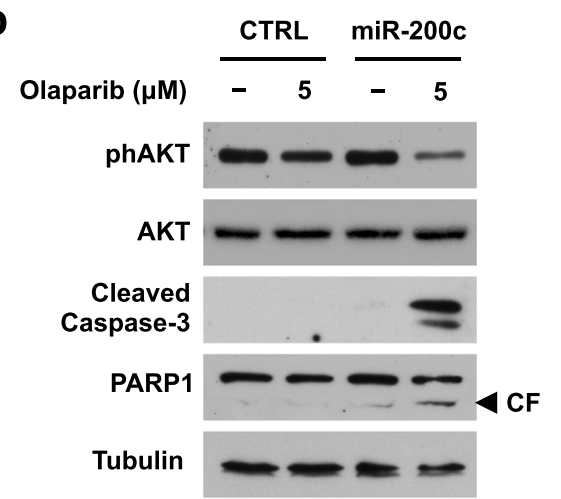

Fig. 8 Effect of miR-200c overexpression on Olaparib-induced apoptosis. SKOV3 cells were stably transfected with a plasmid carrying the precursor of miR-200c (miR-200c) and its corresponding vector control (CTRL), then treated for $144 \mathrm{~h}$ with Olaparib. a) The percentages of early apoptotic and late apoptotic cells were obtained by flow cytometry quadrant analysis with annexin A5 FITC/7-AAD double staining, and expressed as histograms. b) The expression of the apoptosis related proteins phAKT (Ser473), AKT, cleaved Caspase 3 and cleaved PARP1 was determined by Western blot analysis. Tubulin expression was used as internal control. The images are representative of at least two independent experiments. CF, cleaved form of PARP1 


\section{Discussion}

OC represents the fifth principal cause of cancer-related death in women [3]. Its high mortality-to-incidence ratio is essentially due to the absence of OC-specific symptoms and the lack of effective screening strategies. Currently, medical options for OC treatment may include a combination of surgery, chemotherapy and radiation. Nevertheless, the general prognosis in OC patients remains poor, with a 5-year survival rate of about 30\% [4]. It is known that approximately $10-15 \%$ of ovarian cancer patients harbor a germline mutation in genes encoding BRCA1 and BRCA2 proteins, which are involved in the process of homologous recombination (HR) that mediates repair of double stranded DNA breaks [58]. OC patients with BRCA1/2 mutations exhibit impaired ability to repair double-stranded DNA breaks via HR. In this scenario, PARP inhibitors, affecting a second DNA repair pathway, the base excision repair (BER), are able to induce death through a mechanism called synthetic lethality. So, PARPi have been recently approved for treatment of tumors with BRCA1/2 mutations [10]. Nevertheless, the clinical response rates to Olaparib (AZD-2281), a small molecular inhibitor of PARP1, are below 50\% among OC patients with confirmed BRCA1/2 inactivation [59, 60], while a subset of patients without germline BRCA mutations might harbor the so-called "BRCAness" phenotype, an impairment in the HR pathway due to other causes (somatic mutations, as well as epigenetic regulations or mutations in other HR pathways) that could be associated with improved response rate and survival after treatment with these drugs $[11,12]$. At present, one of the major problems of oncologists is the identification of the most appropriate set of patients that could benefit of the treatment with PARPi. In addition, many OC patients develop local recurrence and distant metastases, often accompanied by resistance to both first-line treatments and PARPi $[13,18]$. So, a better understanding of the function of PARP inhibition and the comprehension of drug resistance mechanisms are needed both to predict PARPi clinical efficacy by identifying responsive patients beyond BRCA mutation, and to improve the clinical management of OC patients by introducing novel effective clinical protocols based on combinatorial therapies.

Firstly, we explored the effect of prolonged Olaparib treatment in three OC cell lines and confirmed a differential drug response profile depending on BRCA status. As expected, we observed that treatment with Olaparib is capable of inducing DNA damage and G2/M phase cellcycle arrest in all the $\mathrm{OC}$ cell lines, but such alterations lead to activation of apoptotic pathways only in sensitive UWB cells, maybe due to the persistence of DNA strand breaks, which are restored at $144 \mathrm{~h}$ in UWB-BRCA and SKOV3 cells, with subsequent no apoptosis induction following Olaparib treatment. In agreement with our data, lymphoblastoid cells with mono-allelic mutations of BRCA1 showed a persistent DNA damage upon Olaparib treatment, which rendered them hypersensitive to gamma radiation [61]. However, our results pointed out a partial reduction of clonogenic ability induced by Olaparib in UWB-BRCA cells. Interestingly, in these cells we also observed the activation of autophagy upon Olaparib treatment. It is known that autophagy can be upregulated in response to DNA damage, and in the context of cancer it might function as an adaptive cytoprotective response [62], representing a selective advantage for tumor cells by enhancing drug resistance and aggressiveness, or might act as an alternative to apoptosis to eliminate transformed cells [63-65]. In ovarian cancer cells, this alternative role of autophagy has been suggested to depend on the BRCA status [66]. Our data demonstrates that in the partially resistant UWB-BRCA cells, prolonged Olaparib treatment determines autophagy activation, but fails to stimulate apoptosis. Notably, we found that the autophagy inhibitor chloroquine (CQ) did not induce apoptosis activation in Olaparib-treated UWB-BRCA cells, and reduced the ability of Olaparib to induce apoptosis activation in UWB cells, this indicating an essential role of autophagy in Olaparib citotoxicity in OC cells.

It has been previously demonstrated that NRP1 regulation by various miRNAs plays an important role in mediating tumor growth and angiogenesis [44-47]. Moreover, some previous studies reported NRP1 overexpression in OC with respect to normal ovarian tissue [32] and to benign ovarian tumors [33], as well as a correlation between higher NRP1 expression and shorter survival time [32, 35]. However, there is no full consistency among different studies about the correlation of NRP1 expression with OC hystotype or clinicopathological staging. Interestingly, in our work we observed a lower NRP1 expression in OC with respect to normal ovarian tissues in a total cohort of 40 patients, in which we can find a strong prevalence of serous histotype among the 28 OC samples. Our findings on $\mathrm{OC}$ tissues are consistent with the observation that NRP1 basal levels are lower in OC cell lines derived from serous OC (UWB and UWB-BRCA cells) with respect to those found in cells from peritoneal adenocarcinoma (SKOV3 cells).

Moreover, a comparative analysis of NRP1 expression between pre- and post-chemotherapy $\mathrm{OC}$ samples points out an involvement of NRP1 in OC adaptive response to therapy, this further strengthened by the finding of NRP1 upmodulation in sensitive cells upon prolonged Olaparib treatment. Indeed, we demonstrated that Olaparib could significantly upmodulate NRP1 mRNA and protein only in UWB-BRCA cells, suggesting that in these cells NRP1 expression was sufficient to modulate drug sensitivity, this restricting the effect of Olaparib on cell viability and apoptosis induction. Our data, indicating that NRP1 levels may 
regulate $\mathrm{OC}$ cell lines resistance to $\mathrm{PARPi}$, are in agreement with bioinformatics network analysis suggesting the potential role of NRP1 in drug resistance [36]. Thus, blocking NRP1 expression in OC cells might provide an avenue to increase the sensitivity of drug-resistant cells to Olaparib. Results revealed that downregulation of NRP1 in SKOV3 resistant cells inhibited viability, decreased colony forming potential and induced apoptosis upon Olaparib treatment, indicating that depletion of NRP1 is able to restore sensitivity to PARPi.

Noncoding RNAs, including miRNAs, are endogenous regulatory elements that play a key role in cellular events such as proliferation, differentiation and apoptosis, in both physiological and pathological conditions [39]. In cancer, miRNAs might affect tumor development, progression and drug resistance. Consequently, antagonizing oncogenic miRNAs or restoration of tumor suppressive miRNAs, could represent a reliable tool for improving the cancer therapy [41-43]. However, since each miRNA may regulate several target genes and signaling pathways, miRNA-based treatment requires a careful choice of the potential target.

Increasing evidence show that aberrant expression of miRNAs belonging to the miR-200 family (comprising miR-200a, miR-200b, miR-200c, miR-429 and miR-141) is involved in OC development, as well as in chemoresistance [67-69]. Some of these miRNAs have been shown to interact with NRP1 3'-UTR [54] and to negatively regulate NRP1 signaling [47]. We focused our attention on miR-200c, since it is highly expressed in OC, maybe contributing to epithelial-mesenchymal transition, invasiveness, tumor growth and metastasis [51]. On the other hand, the loss of miR-200c is associated with the acquisition of resistance against various chemotherapeutic agents in different types of cancer, including OC [52]. To date, the function and molecular basis of miR-200c in drug resistance is still undefined. The data obtained about miR-200c expression in our cohort of patients are consistent with previous literature assessing upregulation of miR-200c in OC [48-50], and confirmed an inverse correlation with NRP1 expression.

Our study displayed that overexpression of miR-200c reversed the resistance to Olaparib in OC cells through modulating NRP1 expression. Consistent with our findings, other studies reported a correlation between low expression of miR-200c and paclitaxel resistance in OC [70], and a restoration of paclitaxel sensitivity upon miR-200c overexpression in chemotherapy-resistant cancer cell lines [53, 71, 72] and in a xenograft tumor model [72], this confirming miR-200c as an important control point for the development of chemoresistance in OC.

Our results suggest that tumors with NRP1 downmodulation or miR-200c upmodulation may be more susceptible to PARP inhibition or other strategies based on synthetic lethality. Future studies will determine whether expression levels of NRP1/miR-200c can serve as determinants of therapeutic strategy and of clinical outcome for OC patients.

Indeed, the efficacy in OC cellular models of this novel therapeutic approach, based on the downmodulation of NRP1 through the overexpression of specific miRNA molecules, provides a rationale to translate in vitro experiments in preclinical mouse models. The proposed approach, designed to be administered in combination with PARPi to increase therapeutic efficacy with minimal toxicities, has the potential to develop more effective and less toxic therapeutic protocols for the clinical management of OC patients. Next, we will set up in vivo experiments to test the effects of miR-200c upregulation in overcoming PARPi-related resistance on xenograft mouse models. Future studies will also encompass the role of cancer stem cells (CSCs) in Olaparib resistance mechanisms, by analyzing the efficacy of miR-200c, as monotherapy and in combination with Olaparib, on stemness-related pathways. We think that the development of adjuvant molecular strategies that specifically sensitize CSCs will further contribute to OC eradication.

\section{Conclusions}

In summary, our data describe the PARPi-related response profile of three OC cell lines, clarifying the role of DNA damage, cell cycle arrest and induction of apoptosis/autophagy in PARPi-related resistance, and demonstrate that PARPi sensitivity can be restored by acting on the miR$200 \mathrm{c} / \mathrm{NRP} 1$ axis. In fact, we showed that miR-200c overexpression increases the response of drug resistant OC cells to Olaparib by targeting NRP1, and that both selective inhibition of NRP1 and stable overexpression of miR200c might represent a promising approach to improve Olaparib efficacy. Thus, we think that our study will contribute to the design of novel therapeutic strategies for optimizing the clinical use of PARPi in OC patients.

\section{Additional file}

Additional file 1: Figure S1. Differential effects of Olaparib treatment on cell viability in OC cell lines. Figure S2. Effects of prolonged Olaparib exposure on DNA damage in OC cell lines. Figure S3. Induction of G2/M cell cycle arrest by Olaparib in OC cell lines. Figure S4. Effect of miR-200c overexpression on NRP1. Figure S5. Effect of miR-200c overexpression on autophagy induction

\section{Abbreviations}

7-AAD: 7-amino-actinomycin D; DMSO: Dimethyl sulfoxide; DSBs: Double strand breaks; miRNA: MicroRNA; NRP1: Neuropilin 1; OC: Ovarian cancer; OLA: Olaparib; PARPi: PARP inhibitors; PI: Propidium iodide; siRNA: Small interfering RNA; SSBs: Single strand breaks

\section{Acknowledgements}

The authors are grateful to Dr. Graziella Bernardini for her technical assistance. 


\section{Authors' contributions}

SCe conceived the project. EV, GG, FM, EA, PP, LS and CDV conducted experiments. EV and SCe wrote the manuscript and prepared Figs. EV, FM, EA and SCe analyzed and interpreted the data. SCa, RM and CD provided reagents/materials/analysis tools. ClaM and PBP acquired and managed patients. RM, FR, AA and CM gave comments and reviewed the manuscript. CM and SCe supervised the study. All authors read and approved the final manuscript.

\section{Funding}

This research was conducted with the help of the PRIN 2017 supporting grants (no. ATZ2YK and no. F8ZB89), the Italian Association for Cancer Research (AIRC) grant (no. IG17007) and the Laziolnnova grant 2018 (no. 852017- 13750).

\section{Availability of data and materials}

All data generated during the current study are included within the article.

\section{Ethics approval and consent to participate}

The use of clinical samples of $\mathrm{OC}$ or healthy ovarian tissue complied with the Declaration of Helsinki 1975, revised in 2008, and has been approved by the Institutional Review Board of the Department of Maternal and Child and Urological Sciences of the Sapienza University of Rome. Written consent was obtained from all subjects.

\section{Consent for publication}

Not applicable.

\section{Competing interests}

The authors declare that they have no competing interests.

\section{Author details}

'Department of Experimental Medicine, Sapienza University of Rome, Viale Regina Elena 324, 00161 Rome, Italy. ${ }^{2}$ Department of Clinical and Molecular Medicine, Sapienza University of Rome, 00161 Rome, Italy. ${ }^{3}$ Department of Maternal and Child and Urological Sciences, Sapienza University of Rome, 00161 Rome, Italy. ${ }^{4}$ Department of Public Health and Infectious Diseases, Sapienza University of Rome, 00185 Rome, Italy.

\section{Received: 8 August 2019 Accepted: 25 November 2019}

\section{0.0}

\section{References}

1. Reid BM, Permuth JB, Sellers TA. Epidemiology of ovarian cancer: a review. Cancer Biol Med. 2017;14(1):9-32.

2. Hennessy BT, Coleman RL, Markman M. Ovarian cancer. Lancet. 2009; 374(9698):1371-82

3. Siegel RL, Miller KD, Jemal A. Cancer statistics, 2015. CA Cancer J Clin. 2015; 65(1):5-29.

4. Bookman MA. Optimal primary therapy of ovarian cancer. Ann Oncol. 2016; 27(Suppl 1):i58-62

5. Bergamini A, Pisano C, Di Napoli M, Arenare L, Della Pepa C, Tambaro R, et al. Cisplatin can be safely administered to ovarian cancer patients with hypersensitivity to carboplatin. Gynecol Oncol. 2017;144(1):72-6.

6. Davis A, Tinker AV, Friedlander M. "Platinum resistant" ovarian cancer: what is it, who to treat and how to measure benefit? Gynecol Oncol. 2014;133(3): 624-31

7. Farmer H, McCabe N, Lord CJ, Tutt AN, Johnson DA, Richardson TB, et al. Targeting the DNA repair defect in BRCA mutant cells as a therapeutic strategy. Nature. 2005:434(7035):917-21.

8. Wiltshire TD, Lovejoy CA, Wang T, Xia F, O'Connor MJ, Cortez D. Sensitivity to poly(ADP-ribose) polymerase (PARP) inhibition identifies ubiquitin-specific peptidase 11 (USP11) as a regulator of DNA double-strand break repair. J Biol Chem. 2010;285(19):14565-71.

9. Murai J, Huang SY, Das BB, Renaud A, Zhang Y, Doroshow JH, et al. Trapping of PARP1 and PARP2 by clinical PARP inhibitors. Cancer Res. 2012; 72(21):5588-99.

10. Kaufman B, Shapira-Frommer R, Schmutzler RK, Audeh MW, Friedlander M, Balmaña J, et al. Olaparib monotherapy in patients with advanced cancer and a germline BRCA1/2 mutation. J Clin Oncol. 2015;33(3):244-50.
11. Tan DSP, Rothermundt C, Thomas K, Bancroft E, Eeles R, Shanley S, et al. "BRCAness" syndrome in ovarian cancer: a case-control study describing the clinical features and outcome of patients with epithelial ovarian cancer associated with BRCA1 and BRCA2 mutations. J Clin Oncol. 2008;26(34):5530-6.

12. Turner N, Tutt A, Ashworth A. Hallmarks of "BRCAness" in sporadic cancers. Nat Rev Cancer. 2004:4(10):814-9.

13. Barber LJ, Sandhu S, Chen L, Campbell J, Kozarewa I, Fenwick K, et al. Secondary mutations in BRCA2 associated with clinical resistance to a PARP inhibitor. J Pathol. 2013;229(3):422-9.

14. Johnson N, Johnson SF, Yao W, Li YC, Choi YE, Bernhardy AJ, et al. Stabilization of mutant BRCA1 protein confers PARP inhibitor and platinum resistance. Proc Natl Acad Sci U S A. 2013;110(42):17041-6.

15. Tapodi A, Debreceni B, Hanto K, Bognar Z, Wittmann I, Gallyas F, et al. Pivotal role of Akt activation in mitochondrial protection and cell survival by poly(ADP-ribose)polymerase-1 inhibition in oxidative stress. J Biol Chem. 2005;280(42):35767-75

16. Moskwa P, Buffa FM, Pan Y, Panchakshari R, Gottipati P, Muschel RJ, et al. miR-182-mediated downregulation of BRCA1 impacts DNA repair and sensitivity to PARP inhibitors. Mol Cell. 2011:41(2):210-20.

17. Neijenhuis S, Bajrami I, Miller R, Lord CJ, Ashworth A. Identification of miRNA modulators to PARP inhibitor response. DNA Repair (Amst). 2013 Jun 1;12(6):394-402

18. Choi YE, Meghani K, Brault ME, Leclerc L, He YJ, Day TA, et al. Platinum and PARP inhibitor resistance due to overexpression of MicroRNA-622 in BRCA1mutant ovarian Cancer. Cell Rep. 2016;14(3):429-39.

19. Ellis LM. The role of neuropilins in cancer. Mol Cancer Ther. 2006;5(5):1099107.

20. Hu B, Guo P, Bar-Joseph I, Imanishi Y, Jarzynka MJ, Bogler O, et al. Neuropilin-1 promotes human glioma progression through potentiating the activity of the HGF/SF autocrine pathway. Oncogene. 2007;26(38):5577-86.

21. Lu Y, Xiang H, Liu P, Tong RR, Watts RJ, Koch AW, et al. Identification of circulating neuropilin-1 and dose-dependent elevation following antineuropilin-1 antibody administration. MAbs. 2009;1(4):364-9.

22. Yu DC, Waby JS, Chirakkal H, Staton CA, Corfe BM. Butyrate suppresses expression of neuropilin I in colorectal cell lines through inhibition of Sp1 transactivation. Mol Cancer. 2010:9:276.

23. Neufeld G, Cohen T, Shraga N, Lange T, Kessler O, Herzog Y. The neuropilins: multifunctional semaphorin and VEGF receptors that modulate axon guidance and angiogenesis. Trends Cardiovasc Med. 2002;12(1):13-9.

24. Ohsaka A, Hirota-Komatsu S, Araki M, Komatsu N. Platelet-derived growth factor receptors form complexes with neuropilin-1 during megakaryocytic differentiation of thrombopoietin-dependent UT-7/TPO cells. Biochem Biophys Res Commun. 2015:459(3):443-9.

25. Ceccarelli S, Nodale C, Vescarelli E, Ponteconvi P, Manganelli V, Casella G, et al. Neuropilin 1 mediates keratinocyte growth factor signaling in adipose-derived stem cells: potential involvement in Adipogenesis. Stem Cells Int. 2018;2018:1-18.

26. Hong TM, Chen YL, Wu YY, Yuan A, Chao YC, Chung YC, et al. Targeting neuropilin 1 as an antitumor strategy in lung cancer. Clin Cancer Res. 2007; 13(16):4759-68.

27. Pan Q, Chathery Y, Wu Y, Rathore N, Tong RK, Peale F, et al. Neuropilin-1 binds to VEGF121 and regulates endothelial cell migration and sprouting. J Biol Chem. 2007;282(33):24049-56.

28. Stephenson JM, Banerjee S, Saxena NK, Cherian R, Banerjee SK. Neuropilin-1 is differentially expressed in myoepithelial cells and vascular smooth muscle cells in preneoplastic and neoplastic human breast: a possible marker for the progression of breast cancer. Int J Cancer. 2002;101(5):409-14.

29. Kreuter M, Woelke K, Bieker R, Schliemann C, Steins M, Buechner T, et al. Correlation of neuropilin-1 overexpression to survival in acute myeloid leukemia. Leukemia. 2006;20(11):1950-4

30. Wey JS, Gray MJ, Fan F, Belcheva A, McCarty MF, Stoeltzing O, et al. Overexpression of neuropilin-1 promotes constitutive MAPK signalling and chemoresistance in pancreatic cancer cells. Br J Cancer. 2005;93(2):233-41.

31. Vanveldhuizen PJ, Zulfiqar M, Banerjee $S$, Cherian R, Saxena NK, Rabe A, et al. Differential expression of neuropilin-1 in malignant and benign prostatic stromal tissue. Oncol Rep. 2003;10(5):1067-71.

32. Jiang $H$, Xi Q, Wang F, Sun Z, Huang Z, Qi L. Increased expression of neuropilin 1 is associated with epithelial ovarian carcinoma. Mol Med Rep. 2015:12(2):2114-20.

33. Osada H, Tokunaga T, Nishi M, Hatanaka H, Abe Y, Tsugu A, et al. Overexpression of the Neuropilin 1 (NRP1) gene correlated with poor prognosis in human Glioma. Anticancer Res. 2004;24(2B):547-52. 
34. Baba T, Kariya M, Higuchi T, Mandai M, Matsumura N, Kondoh E, et al. Neuropilin-1 promotes unlimited growth of ovarian cancer by evading contact inhibition. Gynecol Oncol. 2007;105(3):703-11.

35. Siamakpour-Reihani S, Owzar K, Jiang C, Turner T, Deng Y, Bean SM, et al. Prognostic significance of differential expression of angiogenic genes in women with high-grade serous ovarian carcinoma. Gynecol Oncol. 2015;139(1):23-9.

36. Chen C, Hu Y, Li L. NRP1 is targeted by miR-130a and miR-130b, and is associated with multidrug resistance in epithelial ovarian cancer based on integrated gene network analysis. Mol Med Rep. 2016;13(1):188-96.

37. Filipowicz W, Bhattacharyya SN, Sonenberg N. Mechanisms of posttranscriptional regulation by microRNAs: are the answers in sight? Nat Rev Genet. 2008;9(2):102-14.

38. Bartel DP. MicroRNAs: genomics, biogenesis, mechanism, and function. Cell. 2004;116(2):281-97.

39. Jansson MD, Lund AH. MicroRNA and cancer. Mol Oncol. 2012;6(6):590-610.

40. Anastasiadou E, Faggioni A, Trivedi P, Slack FJ. The nefarious nexus of noncoding RNAs in cancer. Int J Mol Sci. 2018;19(7):2072.

41. Szafranska AE, Davison TS, John J, Cannon T, Sipos B, Maghnouj A, et al. MicroRNA expression alterations are linked to tumorigenesis and nonneoplastic processes in pancreatic ductal adenocarcinoma. Oncogene. 2007; 26(30):4442-52.

42. Hayes J, Peruzzi PP, Lawler S. MicroRNAs in cancer: biomarkers, functions and therapy. Trends Mol Med. 2014;20(8):460-9.

43. Bader AG, Brown D, Winkler M. The promise of microRNA replacement therapy. Cancer Res. 2010;70(18):7027-30.

44. Liu Q, Xu Y, Wei S, Gao W, Chen L, Zhou T, et al. miRNA-148b suppresses hepatic cancer stem cell by targeting neuropilin-1. Biosci Rep. 2015;35(4): e00229.

45. Zhang G, Chen L, Khan AA, Li B, Gu B, Lin F, et al. miRNA-124-3p/neuropilin1(NRP-1) axis plays an important role in mediating glioblastoma growth and angiogenesis. Int J Cancer. 2018;143(3):635-44

46. Zhu H, Jiang $X$, Zhou $X$, Dong $X$, Xie $K$, Yang $C$, et al. Neuropilin-1 regulated by miR-320 contributes to the growth and metastasis of cholangiocarcinoma cells. Liver Int. 2018;38(1):125-35.

47. Ma L, Zhai B, Zhu H, Li W, Jiang W, Lei L, et al. The miR-141/neuropilin-1 axis is associated with the clinicopathology and contributes to the growth and metastasis of pancreatic cancer. Cancer Cell Int. 2019;19(1):1-15.

48. Iorio MV, Visone R, Di Leva G, Donati V, Petrocca F, Casalini P, et al. MicroRNA signatures in human ovarian cancer. Cancer Res. 2007;67(18): 8699-707.

49. Kan CWS, Hahn MA, Gard GB, Maidens J, Huh JY, Marsh DJ, et al. Elevated levels of circulating microRNA-200 family members correlate with serous epithelial ovarian cancer. BMC Cancer. 2012;12:627.

50. Cao Q, Lu K, Dai S, Hu Y, Fan W. Clinicopathological and prognostic implications of the miR-200 family in patients with epithelial ovarian cancer. Int J Clin Exp Pathol. 2014;7(5):2392-401.

51. Sulaiman SA, Ab Mutalib N-S, Jamal R. miR-200c Regulation of Metastases in Ovarian Cancer: Potential Role in Epithelial and Mesenchymal Transition. Front Pharmacol. 2016;7:271.

52. Mutlu M, Raza U, Saatci Ö, Eyüpoğlu E, Yurdusev E, Şahin Ö. miR-200c: a versatile watchdog in cancer progression, EMT, and drug resistance. J Mol Med. 2016;94(6):629-44.

53. Cochrane DR, Howe EN, Spoelstra NS, Richer JK. Loss of miR-200c: a marker of aggressiveness and Chemoresistance in female reproductive cancers. J Oncol. 2010:2010:821717.

54. Lin CH, Jackson AL, Guo J, Linsley PS, Eisenman RN. Myc-regulated microRNAs attenuate embryonic stem cell differentiation. EMBO J. 2009;28(20):3157-70

55. Ceccarelli S, D'Amici S, Vescarelli E, Coluccio P, Matricardi P, di Gioia C, et al. Topical KGF treatment as a therapeutic strategy for vaginal atrophy in a model of ovariectomized mice. J Cell Mol Med. 2014;18(9):1895-907.

56. Rotolo S, Ceccarelli S, Romano F, Frati L, Marchese C, Angeloni A. Silencing of keratinocyte growth factor receptor restores 5-fluorouracil and Tamoxifen efficacy on responsive Cancer cells. PLoS One. 2008;3(6):e2528.

57. D'Amici S, Ceccarelli S, Vescarelli E, Romano F, Frati L, Marchese C, et al. TNF alpha modulates fibroblast growth factor receptor 2 gene expression through the pRB/E2F1 pathway: identification of a non-canonical E2F binding motif. PLoS One. 2013;8(4):e61491.

58. Li AJ, Karlan BY. Genetic factors in ovarian carcinoma. Curr Oncol Rep. 2001; 3(1):27-32.

59. Audeh MW, Carmichael J, Penson RT, Friedlander M, Powell B, Bell-McGuinn $\mathrm{KM}$, et al. Oral poly(ADP-ribose) polymerase inhibitor olaparib in patients with BRCA1 or BRCA2 mutations and recurrent ovarian cancer: a proof-ofconcept trial. Lancet. 2010;376(9737):245-51.

60. Fong PC, Yap TA, Boss DS, Carden CP, Mergui-Roelvink M, Gourley C, et al. Poly(ADP)-ribose polymerase inhibition: frequent durable responses in BRCA carrier ovarian cancer correlating with platinum-free interval. J Clin Oncol. 2010;28(15):2512-9.

61. Bourton EC, Ahorner PA, Plowman PN, Zahir SA, Al-Ali H, Parris CN. The PARP-1 inhibitor Olaparib suppresses BRCA1 protein levels, increases apoptosis and causes radiation hypersensitivity in BRCA1+/lymphoblastoid cells. J Cancer. 2017:8(19):4048-56.

62. Rodriguez-Rocha H, Garcia-Garcia A, Panayiotidis MI, Franco R. DNA damage and autophagy. Mutat Res. 2011 Jun;711(1-2):158-66.

63. Liu C, Yan X, Wang H-Q, Gao Y-Y, Liu J, Hu Z, et al. Autophagy-independent enhancing effects of Beclin 1 on cytotoxicity of ovarian cancer cells mediated by proteasome inhibitors. BMC Cancer. 2012;12:622

64. Levine B, Yuan J. Autophagy in cell death: an innocent convict? J Clin Invest. 2005;115(10):2679-88.

65. Opipari AW Jr, Tan L, Aurora A, Liu JR, Boitano AE, Sorenson DR. Resveratrolinduced autophagocytosis in ovarian Cancer cells. Cancer Res. 2004;64(2): 696-703.

66. Wan B, Dai L, Wang L, Zhang Y, Huang H, Qian G, et al. Knockdown of BRCA2 enhances cisplatin and cisplatin-induced autophagy in ovarian cancer cells. Endocr Relat Cancer. 2018;25(1):69-82.

67. Banno K, Yanokura M, lida M, Adachi M, Nakamura K, Nogami Y, et al. Application of microRNA in diagnosis and treatment of ovarian cancer. Biomed Res Int. 2014:2014:232817.

68. Muralidhar GG, Barbolina MV. The miR-200 family: versatile players in epithelial ovarian cancer. Int J Mol Sci. 2015;16(8):16833-47.

69. Chen SN, Chang R, Te Lin L, Chern CU, Tsai HW, Wen ZH, et al. MicroRNA in ovarian cancer: biology, pathogenesis, and therapeutic opportunities. Int $J$ Environ Res Public Health. 2019:16(9):1-14.

70. Marchini S, Cavalieri D, Fruscio R, Calura E, Garavaglia D, Nerini IF, et al. Association between miR-200c and the survival of patients with stage I epithelial ovarian cancer: a retrospective study of two independent tumour tissue collections. Lancet Oncol. 2011;12(3):273-85.

71. Cochrane DR, Spoelstra NS, Howe EN, Nordeen SK, Richer JK. MicroRNA200c mitigates invasiveness and restores sensitivity to microtubule-targeting chemotherapeutic agents. Mol Cancer Ther. 2009;8(5):1055-66.

72. Cittelly DM, Dimitrova I, Howe EN, Cochrane DR, Jean A, Spoelstra NS, et al. Restoration of miR-200c to ovarian Cancer reduces tumor burden and increases sensitivity to paclitaxel. Mol Cancer Ther. 2012;11(12):2556-65.

\section{Publisher's Note}

Springer Nature remains neutral with regard to jurisdictional claims in published maps and institutional affiliations.

Ready to submit your research? Choose BMC and benefit from:

- fast, convenient online submission

- thorough peer review by experienced researchers in your field

- rapid publication on acceptance

- support for research data, including large and complex data types

- gold Open Access which fosters wider collaboration and increased citations

- maximum visibility for your research: over $100 \mathrm{M}$ website views per year

At BMC, research is always in progress.

Learn more biomedcentral.com/submissions 